\title{
Wigner crystallization of two-dimensional electron systems on solid $\mathbf{H}_{2}$-films
}

\author{
F. MUGELE and P. LEIDERER
}

Universität Konstanz, Fakultät für Physik, 7750 Konstanz, Germany

\begin{abstract}
We have investigated the transport properties of two-dimensional electron systems (2DES) on quench-condensed hydrogen films for various electron densities up to $n_{e}=1.5 x^{10} \mathrm{~cm}^{-2}$. In the range of the plasma parameter $\Gamma=\left\langle V_{\text {coul. }}>/\left\langle E_{\text {kin }}>\right.\right.$ from 40 to 180 we have observed a behavior $\ln \mu x \Gamma$ of the electron mobility $\mu$ with a distinct transition from a smaller slope for $\Gamma<125$ to a steeper one for $\Gamma 125$. This signature is interpreted as the Wigner crystallization of the 2DES.
\end{abstract}

\section{Introduction}

Since the first observation of the Wigner transition by Grimes and Adams [1] in 1979 2DES on cryogenic surfaces such as helium (bulk and saturated films), hydrogen and neon have attracted continuous attention Beside the use of 2 DES as surface probes $[2,3]$ these investigations have explored the phase diagram of 2DES over a wide range of electron densities $[4,5]$. In our experiments we use thin solid hydrogen films as a substrate. The mobilities we reach are comparable to those other authors obtain on $\mathrm{H}_{2}$ - or Ne-crystals. In this paper we report on the observation of electron correlations in 2DES on these solid hydrogen films.

\section{Experimental}

We prepared $\approx 3 \mu \mathrm{m}$ thick hydrogen films by quench-condensation onto a glass plate at $1.7 \mathrm{~K}$. Before charging the hydrogen surface a small amount of helium gas was admitted to the cell in order to slow down the electrons and thus prevent them from penetrating the film. The electron density was proportional to the applied electric holding field. We measured the conductivity of the 2DES with the Sommer-Tannertechnique [6] at a frequency of $50 \mathrm{kHz}$. Initially the electron conductivity varied strongly in the temperature range between $1.7 \mathrm{~K}$ and $4.2 \mathrm{~K}$ due to trapping of the electrons at surface defects. By thermal annealing we obtained very smooth hydrogen films on which - for the lowest electron densities investigated - the conductivity was found to be almost constant over the whole temperature range. Details of the experimental setup and the annealing procedure are given in refs. 7 and 3 .

\section{Results}

Fig. 1 shows the conductivity vs. temperature for different electron densities between $3.4 \times 10^{8} \mathrm{~cm}^{-2}$ and $1.5 \times 10^{10} \mathrm{~cm}^{-2}$. All the curves were taken on the same hydrogen film with a constant helium gas density of $4 \times 10^{19} \mathrm{~cm}^{-3}$ in the cell. (Localization of electrons in the helium gas atmosphere can be excluded at these low He densities.) For the lowest electron densities (curve $a$ and $b$ ) the conductivity is essentially constant over the whole temperature range except for a small modulation and a slight overall increase which are both due to the adsorption of He monolayers on the hydrogen surface [2]. Especially the decrease of the conductivity between $3.3 \mathrm{~K}$ and $4.2 \mathrm{~K}$ which is observed in all the curves is caused by the scattering of the electrons from the density fluctuations within the first He monolayer.

For higher electron densities a decrease of up to three orders of magnitude toward low temperatures is observed. Furthermore, at fixed temperature the conductivity does not follow the simple relation $\dot{\sigma}=\mathrm{n}_{c} \mathrm{e}$ with a constant average mobility $\mu$ : $\mu$ always decreases upon increasing the electron density. We attribute 


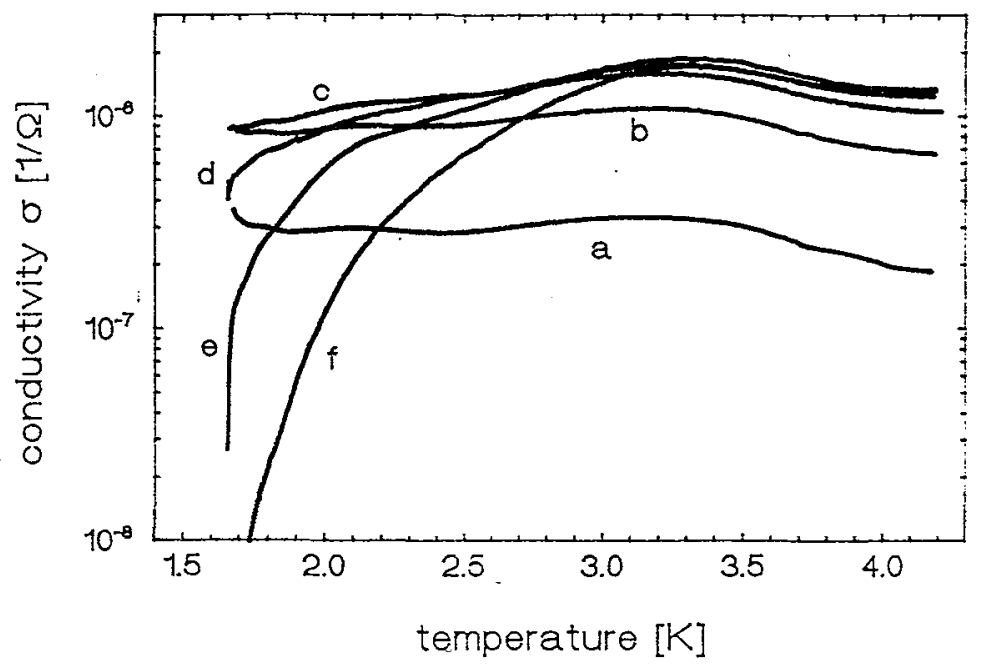

Fig. 1 Conductivity $\sigma$ vs temperature $T$ for different electron densities $n_{e}$; a) $n_{e}=3.4$; ) $n_{e}=12$; $) n_{e}=29$;) $n_{e}=48$; e) $n_{e}=85:$ f) $n_{e}=150$; in $10^{8} \mathrm{~cm}^{-2}$.

this to Coulomb interaction in combination with localization of individual electrons at surface heterogenities.

A more detailed analysis of the data requires the compensation of the effect of adsorbed helium. Although being very interesting in itself $[2,3]$ it complicates the interpretation of the data with regard to the interaction between the electrons. At $4.2 \mathrm{~K}$ the electrons see a hydrogen surface which is covered by about half a monolayer of adsorbed helium. At the low temperature end of our measurements the $\mathrm{H}_{2}$-film is covered with more than three monolayers of helium. Therefore the electrons are lifted off by about $10 \AA$ from the hydrogen surface with its irregularities. At the same time they are further extended perpendicular to the surface because the adsorbed helium reduces the effective polarizability of the substrate.

Fig. 2a shows the mobility derived from the data in Fig. 1 versus the plasma parameter $\Gamma=e^{2}\left(\pi n_{e}\right)^{1 / 2} /\left(\varepsilon \mathrm{k}_{\mathrm{B}} \mathrm{T}\right)$ (e: electronic charge; $\bar{\varepsilon}=1.15$ : effective dielectric constant for bare $\mathrm{H}_{2}$ ) at different fixed temperatures, i.e. at different fixed helium coverages. For $\Gamma<110$ the data essentially collaps on parallel straight lines in this logarithmic plot. The systematic shift to higher mobilities at lower temperatures is due to the adsorbed helium. At higher values of $\Gamma$ a smeared bending down of the mobility can be seen. In Fig. $2 b$ the helium effect is compensated by shifting the parallel parts of the curves below $\Gamma$ $=110$ one upon another. Now a transition from a weaker to a stronger dependence of the mobility on $\Gamma$ becomes apparent around $\Gamma=125$. We attribute this to the Wigner crystallization of the 2DES. This interpretation is supported by the second information beside the conductivity, one can derive from Sommer-Tanner type measurements [8]: The data yield also a value for the capacity between the measuring electrodes and the 2DES at the $\mathrm{H}_{2}$-surface. In Fig. 3 this capacity which is related to the number of mobile electrons contributing to the signal is plotted vs temperature. While the apparent capacity remains constant over the whole temperature range for the lower electron densities (curve $b$ and $c$ ) a clear transition from a high temperature to a low temperature state is observed for the higher electron densities (curve $d, e, t)$. The transition temperature $T_{c}$ increases with ne and the transition itself is broadended for higher $T$. The values of $\Gamma_{c}$ derived from $T_{c}$ are $107,118,128$ for $n_{e}=4.8 \times 10^{9} \mathrm{~cm}^{-2}(d), n_{e}=8.5 \times 10^{9} \mathrm{~cm}^{-2}(e)$ and $n_{e}=1.5 \times 10^{10} \mathrm{~cm}^{-2}(f)$ respectively. The arrows in Fig. 1 mark the respective $T_{c}$ in the conductivity curves. In curve $d$ and e one can make out a change in the slope at the respective critical temperature. In curve $f$ the effect is completely obscured by thermal disorder. A very similar behavior has been reported by Kajita for a $2 \mathrm{DES}$ on solid Ne [5]. 

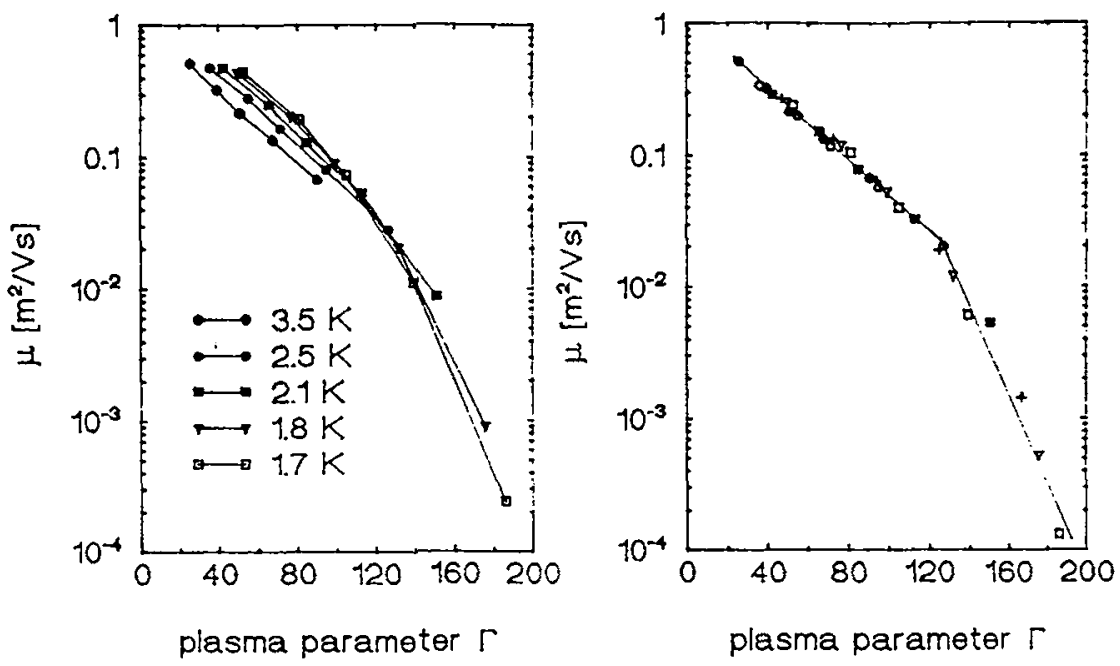

Fig. 2 Mobility $\mu$ derived from curves $b$ ) to $f$ in Fig. 1 vs plasma parameter $\Gamma$ at difierent fixed temperatures. a) before b) after compensation of the influence of adsorbed He-monolayers.

\section{Discussion}

The 2DES is exposed to several random potentials. First electrons are scattered by the helium gas atoms. Since the gas atom density is nearly constant in our case, this leads to a temperature independent scattering rate [9] which limits the electron mobility in curve a of Fig. 1. Second our hydrogen surface was certainly not completely flat, although being much smoother than previously investigated films $[3,7]$, where the localization of electrons at surface inhomogenities plays a major role already at low $n_{\mathrm{c}}$. For higher electron densities, which are accompanied by larger electric holding fields, surface roughness becomes a more effective single electron pinning center. Localized electrons then modify the potential landscape of the hydrogen surface seen by the other electrons. The bebavior $\ln \mu \propto \Gamma$ shown in Fig. 2 for $\Gamma<125$ which we have already observed previously [10] led us to the conclusion that Coulomb interaction $\langle V\rangle \times n_{e} n^{1 / 2}$ with pinned electrons is the dominant reason for the reduction of electron mobility in the fluid phase of the 2DES.

When Wigner crystallization takes place increasing correlations among the electrons enhance the effectiveness of the pinning centers. Aready a few pinning centers can suppress the mobility of the whole $2 D E S$. At the same time the number of mobile electrons is reduced and thus the apparent capacity decreases.

The structure of the Wigner phase and the transport mechanism in this regime remain unclear at this stage. As the 2DES is exposed to a strong random potential from pinned electrons the formation of one welldefined Wigner crystal is rather unlikely. Many small distorted crystallites which can move individually might explain the otherwise surprisingly small kink in the mobility at the transition. At lower temperatures more of these crystallites are pinned and the transition is more marked (cf. arrows in Fig. 1). This behavior agrees qualitatively with Kajita's results on solid $\mathrm{Ne}[5]$.

The difference of about $20 \%$ between $\Gamma_{c}$ at $n_{e}=4.8 \times 10^{9} \mathrm{~cm}^{-2}$ and $n_{e}=1.5 \times 10^{10} \mathrm{~cm}^{-2}$ is not completely understood. First order quantum statistical corrections to the kinetic energy can only account for a difference of $2 \%$ between these electron densities:

with $\mathrm{T}_{\text {Fermi }}=2.7 \times 10^{-11} \mathrm{~K}\left(\mathrm{n}_{\mathrm{e}} \times \mathrm{cm}^{2}\right)$.

$$
<E_{k i n}>=k_{B} T\left(1-0.25 T_{\text {Fermi }} / T\right) \text {, }
$$




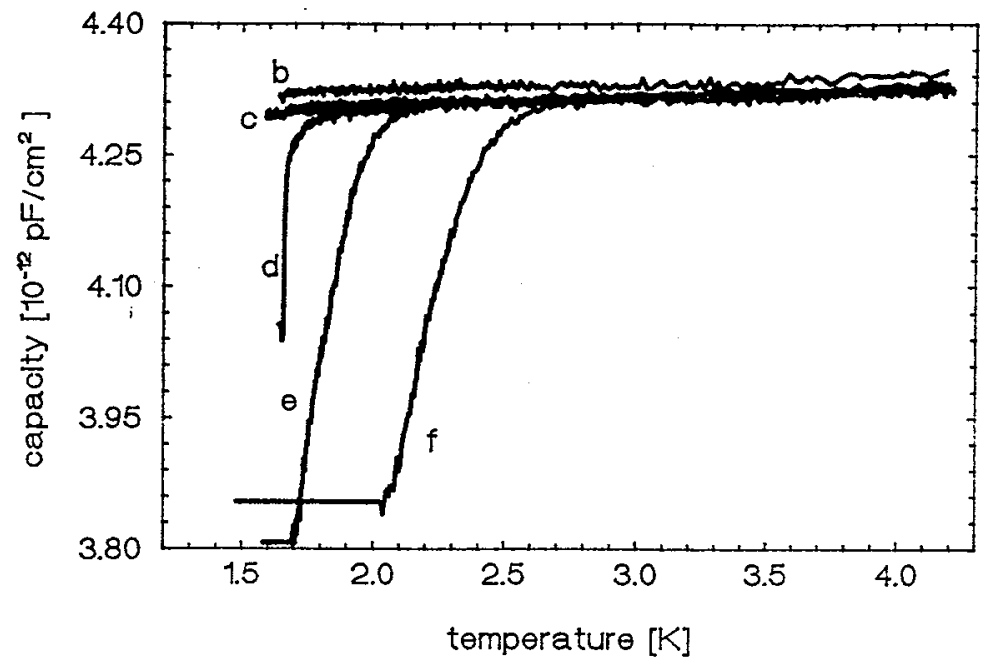

Fig. 3 Capacity vs temperature for the data of curves b) to f) in Fig. 1.

The underestimation of Coulomb interaction at the transition temperature of $1.7 \mathrm{~K}$ where the substrate screening expressed by $\bar{\varepsilon}$ is reduced by three or four monolayers of adsorbed helium explains another $2 \%$ or $3 \%$. Thus both effects are too weak to explain the whole difference.

\section{Conclusions}

We have measured the temperature-dependent conductivity of a 2DES on quench-condensed sulid hydrogen films. We find that the mobility is determined by the interaction between pinned and mobile electrons in a range of $\Gamma$ from 40 to 110 . At $\Gamma$ around 125 a transition to a highly correlated Wigner state occurs. A difference between the critical values of $\Gamma$ at lower and at higher electron densities remains unexplained. The investigated electron densities were still too low to observe quantum statistical corrections. Technical modifications of the experimental setup in order to reach the necessary densities are underway.

\section{References:}

[1] GRMES C.G. and ADAMS G., Phys. Rev. Lett. 42 (1979) 795.

[2] CIESLIKOWSKI D., DAHM A.J. and LEIDERER P., Phys. Rev. Lett. 58 (1987) 1751.

[3] ALBRECHT U., LEDERER P. and KONO K., Surf. Sci. 283 (1993) 423.

[4] STAN M.A. and DAHMM A.J., Phys. Rev. B40 (1989) 8995.

[5] KAJITA K., J. Phys. Soc. Japan 54 (1985) 4092.

[6] SOMMER W.T. and TANNER D.J., Phys. Rev. Lett. 20 (1971) 1751.

[7] KONO K., ALBRECHT U. and LEIDERER P., J. Low Temp. Phys. 82 (1991) 279.

[8] MEHROTRA R. and DAHM A.J., J. Low Temp. Phys. 67 (1987) 115.

[9] SAITOH M., J. Phys. Soc. Japan 42 (1977) 201.

[10] MUGELE F., ALBRECHT U., LEIDERER P. and KONO K., J. Low Temp. Phys. 89 (1992) 743. 